\title{
RECOGNITION OF SINGLE AND OVERLAY OF OBJECTS ON A CONVEYOR BELT
}

\author{
S.V. Savicheva ${ }^{a}$
}

${ }^{a}$ Murom Institute (branch) or the Federal State Budget Institution of Higher Professional Education "Vladimir State University Alexander G. and Nicholai G. Stoletovs”, Russia - savicheva.svetlana@ gmail.com

KEY WORDS: Recognition, Flat object, Binary image, Circuit, Curvature, Signature, Sign.

\begin{abstract}
:
Proposed a method for detection of flat objects when they overlap condition. The method is based on two separate recognition algorithms flat objects. The first algorithm is based on a binary image of the signature of the object plane. The second algorithm is based on the values of the discrete points in the curvature contour of a binary image. The results of experimental studies of algorithms and a method of recognition of individual superimposed flat objects.
\end{abstract}

\section{MANUSCRIPT}

When sorting and control parts, assembling products, which are, for example, on an assembly line, the problem of recognition of individual and superimposed objects [1,2].

If the problem of recognition of individual objects already well solved [3], the question of recognition of individual objects as they imposed a state yet to be resolved.

Development of algorithms for recognizing objects overlap several research centers in Japan and the United States [4]. However, not algorithms and methods suitable for practical use. In Russia, research in this area is not yet underway.

In this regard, the development and practical application of new algorithms for fast and accurate recognition of individual and superimposed objects is an urgent research task that would create an industrial system of automatic recognition.

In this paper, we consider two separate recognition algorithm and a method for recognition of superimposed (two) flat objects.

Under the flat object refers to real objects, products, details of height $\mathrm{h}$ is much smaller than its length.

\section{RECOGNITION ALGORITHMS INDIVIDUAL FLAT OBJECTS}

The first algorithm is based on the use of signatures of the binary object image plane.

At the stage of learning implements the following steps:

1. Generation of 2000 options for the location of each flat object in the field of view of the system.

Basic information (parameters) for the generation of options for the location of flat objects are the coordinates of the center of gravity $x_{u}$ and $y_{u}$ and angle of the objects. The parameter values are specified using the random number. The values of the angle change in the range from 0 to 360 degrees (one step is equal degrees), and the coordinates $x_{u}$ and $y_{u}$ within the $x_{1}<x_{\mathrm{L}}<x_{2} ; y_{1}<y_{\mathrm{L}}<y_{2}$, where $\left(x_{1} ; y_{1}\right), \quad\left(x_{2} ; y_{2}\right)$ - the coordinates of the diagonal of the image, which should not go for the redistribution of the field, the size $1023 \times 1023$. For research, the size of the working area was chosen to $1024 \times 1024$ pixels. The size of the workspace is $1023 \times 1023$ points. 20000 variants is generated separate flat objects 10 (Figure 1). They are placed in the database.
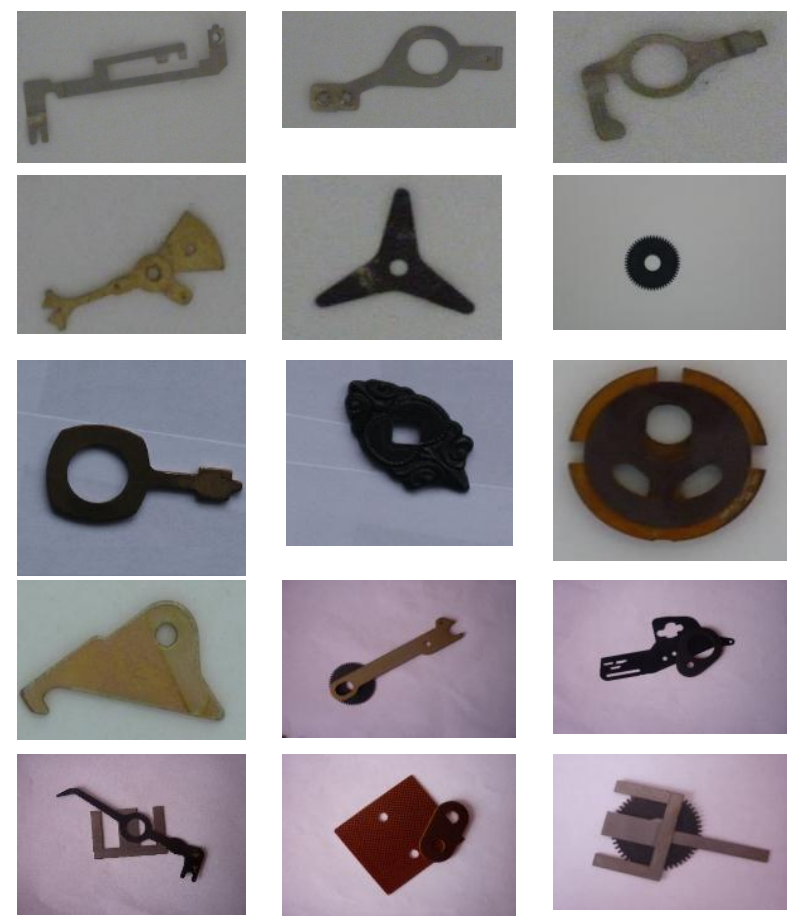

Figure 1. Examples of individual and superimposed flat objects

2. Determination of the frequency of occurrence of each variant location in the field of vision recognition systems. The results are also placed in the database

3. Pretreatment

The proposed method of image pre-processing of flat objects comprises the following steps [4]: 1) reduction of the noise (mostly background) on the image of the object to the desired; 2) binarization of images; 3) marking objects in the image; 4) removing the objects from the memory of non-integer; 5) edge detection of binary images of objects; 6) determination of the center of gravity of binary images.

Results of operations 5 and 6, the provisional image processing flat objects stored in the database.

4. Formation of R-functions for each binary image object

The algorithm is used as an indication of the function $R\left(r_{i}\right)$. It represents a set of values $\left\{r_{1}\right\}$ distances from the center of the object to the points of its outer contour. This function will be called R-function. 
The process of forming an R-function binary image on its contour is shown in Figure 2.

Formation of R-function object is to arrange an array with values $r_{\mathrm{i}}(i=1,2,3, \ldots, m)$ in the order they are defined in the bypass circuit.

It should be noted that, when the R-specs, its first element $r 1$ is the distance from the center point to the first circuit, the first found during the scanning of the image field from left to right and top to bottom. R-functions of all 20,000 images of objects 10 are entered into the database.

Figure 2 shows examples of $\mathrm{R}$-functions of certain objects $(1,2$, $3,4)$ in Figure 1.

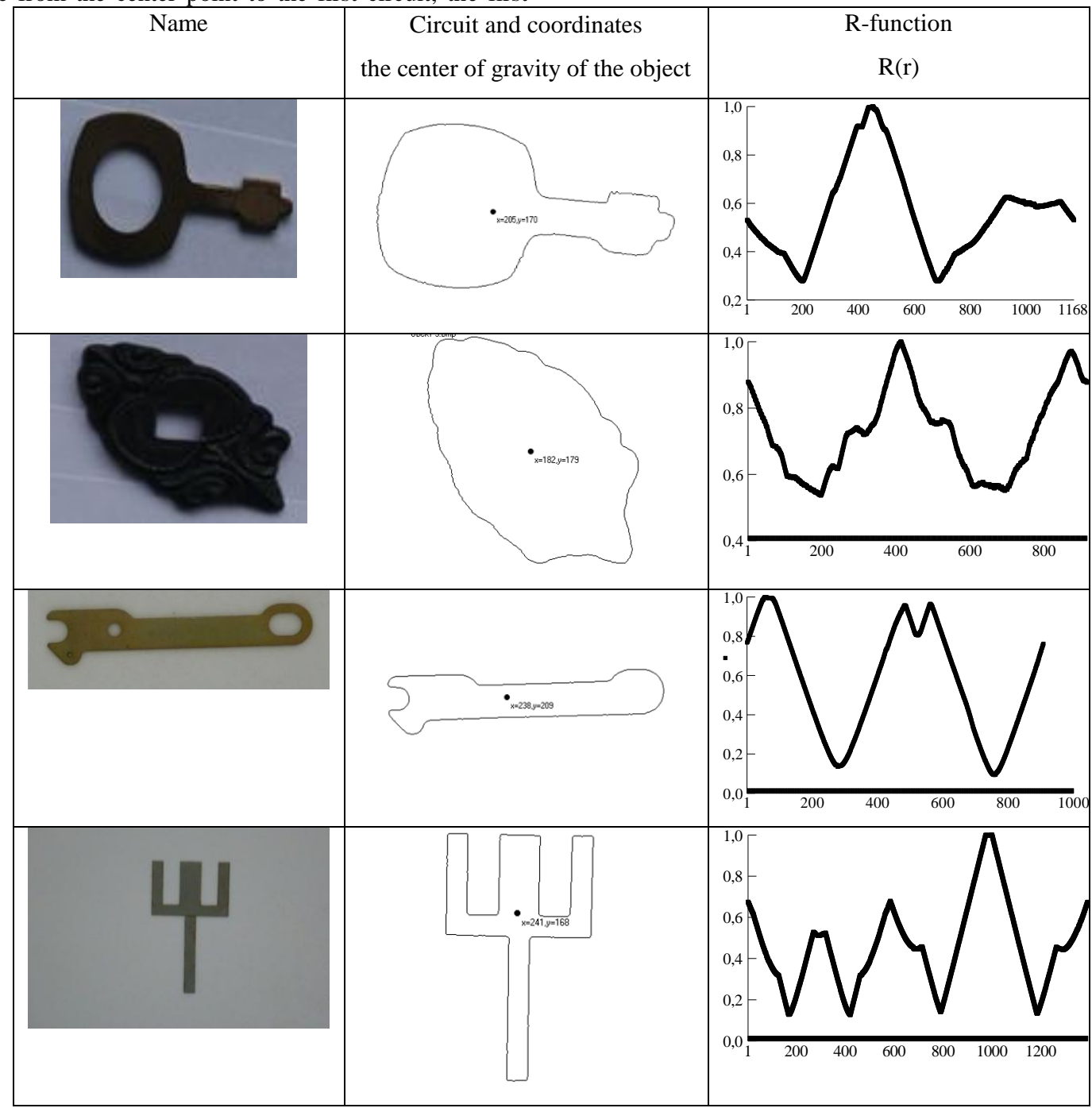

Figure 2. Charts R-functions of some real flat objects

2. Recognition of planar objects and determining the required number of standards

In recognition of its object R-function compared with standard rs-functionality of objects based on the following expression

$$
Z_{k l}=\sqrt{\frac{1}{n+1} \sum_{i=1}^{n}\left(r_{i k}-r_{i l}\right)^{2}}
$$

where

$k$ - number of the reference object;

$l$ - number of recognizable object;

$r_{i k}=\left\{r_{i}(k)\right.$, if i $i \leq m_{k} ; 0$ - otherwise $\}$;

$r_{i l}=\left\{r_{i}(l)\right.$, if $i \leq m i ; 0$ - otherwise $\}$;

$m_{k}, m_{l}$ - the number of R-function number $k$ and the number of recognizable object $l$, respectively;

$Z_{k l}$ - the standard deviation of the values of Rfunctions identify objects with a number of reference $l$ rsfunction with $k$.

$$
n=\max _{k, l}\left\{m_{k}, m_{l}\right\} \text {. }
$$

The values of the missing elements, compared R- and Refunctions assumed to be zero. Unlike $m_{k}$ and $m_{l}$ due to the fact that different objects have different number of contour elements.

The decision to identify a recognizable object is made based on the following expression:

$$
\min _{k, l} Z_{k l}^{O}=\min _{k}\left\{\min _{l} Z_{k l}\right\}
$$

where

$$
\min Z_{k l}\left\{{ }^{*}\right\} \text { - minimum value } Z^{\prime} k l \text { among }
$$

all $Z^{\prime} k l$ for $\mathrm{k}=1,2, \ldots, \mathrm{N}$ and the constant value $l$;

Studies have been conducted with all versions 2000010 separate images of flat objects with the selected amount of standards. 
Studies have confirmed the results obtained during the training phase. Figure. 5 and Figure. 6 are graphs of the probability of correct recognition and time on all 10 original objects on the number of plane standards.

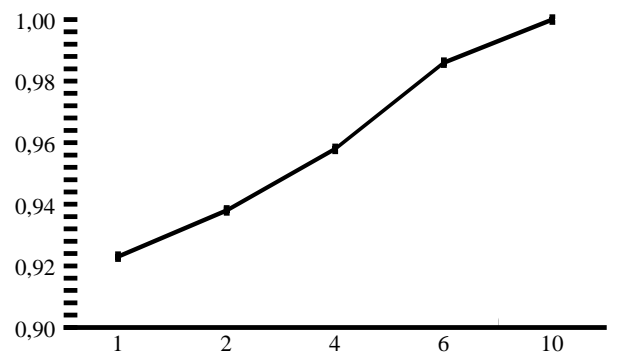

Figure 5. A plot of the probability of correct recognition of a separate flat object on the number of standards

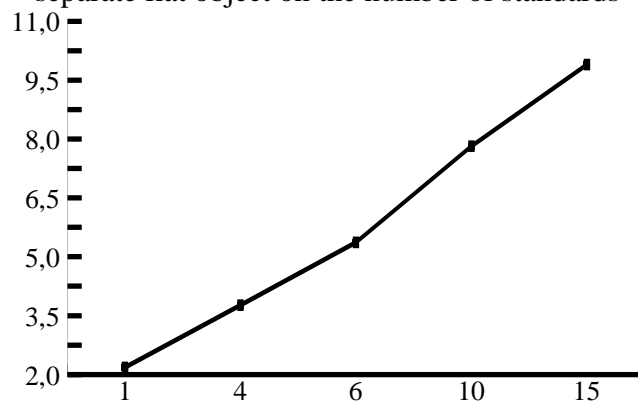

Figure. 6. Schedule time change detection separate flat object on the number of standards
Analysis of the results shows that the detection accuracy of certain embodiments of real planar object using standards of 10 to $100 \%$ and further increase the number of reference only leads to increase in the recognition time for each object.

The second algorithm is based on the values of the discrete points in the curvature contour of a binary image of a flat object.

All the stages and steps of the algorithm are almost similar to the steps and the first steps of the algorithm.

The difference function is formed from the curvature values at discrete points of the binary contour image of the object plane. Therefore, the process of determining the values of a curvature of the contour and curvature of the shaping function will be called A-function.

For one-point for seamless contours of binary images of flat objects used algorithm, which forms a discrete contour line of 4 and d-connected points

This means a single-point circuit consists of points, the curvature of which can take only three values $-0^{\circ}, 90^{\circ}$ and $135^{\circ}$. Therefore, the determination of the value of curvature at such a circuit can be done in a logical way with masks, which greatly simplifies and accelerates the process of formation of signs as opposed to analytical algorithms for computing curvature points to the discrete circuit.

In order to determine the curvature at points one-element discrete line 16 , it is sufficient to scan the mask size of $3 \times 3$ elements.

In Figure 7 shows examples of normalized A-functions of certain objects (1-4) of Figure 1.

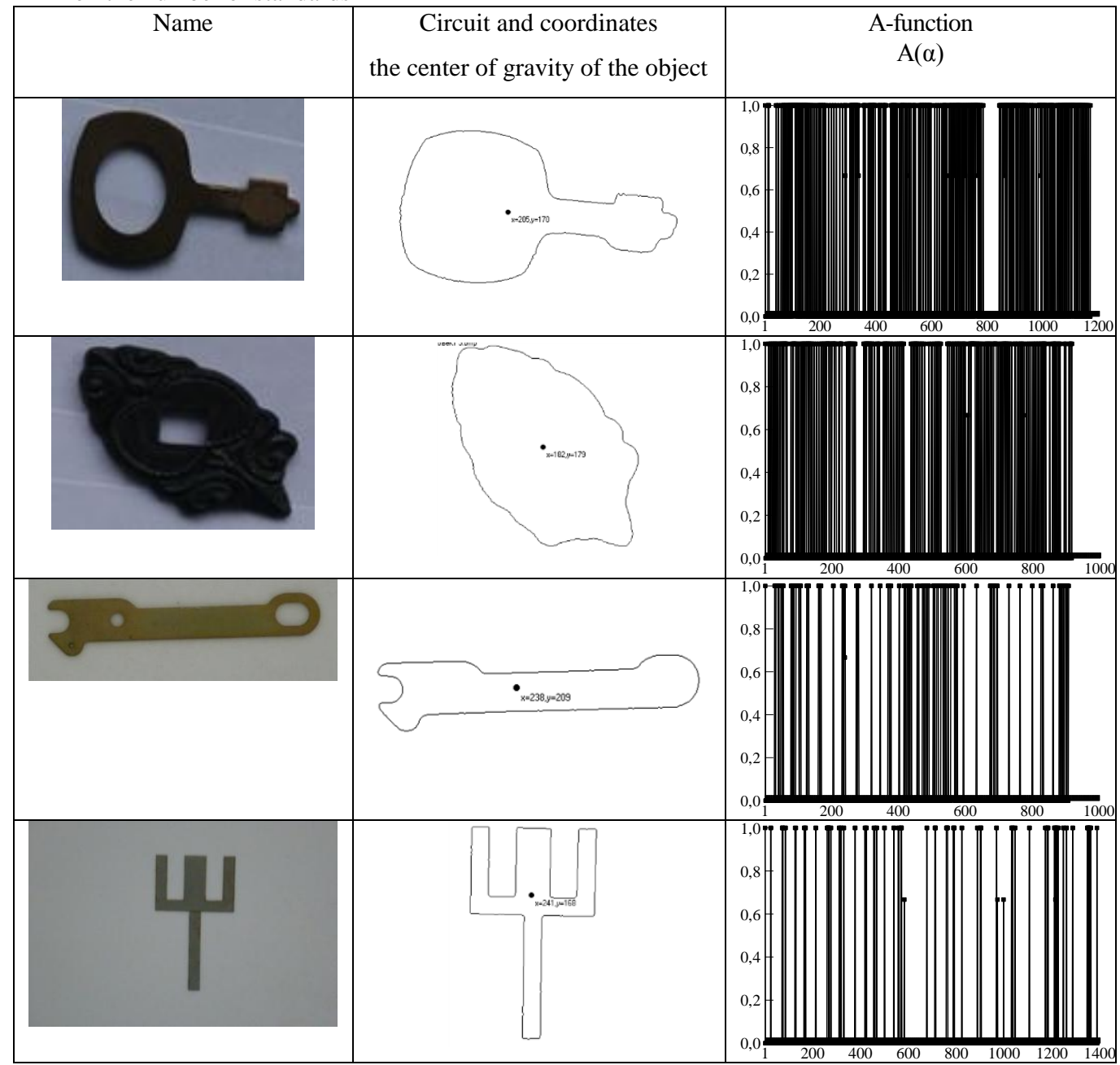

Figure. 7. Graphs and functions of some specific real flat objects 
Figure. 8 and Figure 9 are plots of the probability of correct recognition and time on all 10 original flat objects on the amount of standards.

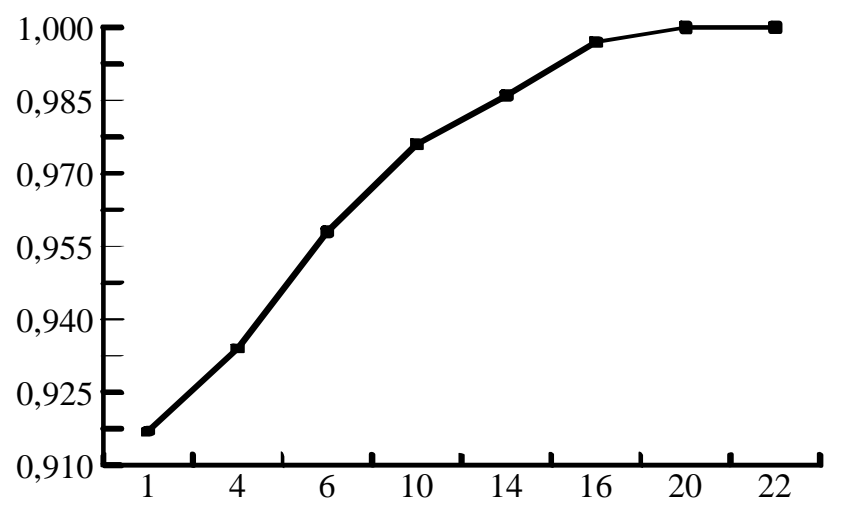

Figure. 8. A plot of the probability of correct recognition of individual flat objects on the number of standards

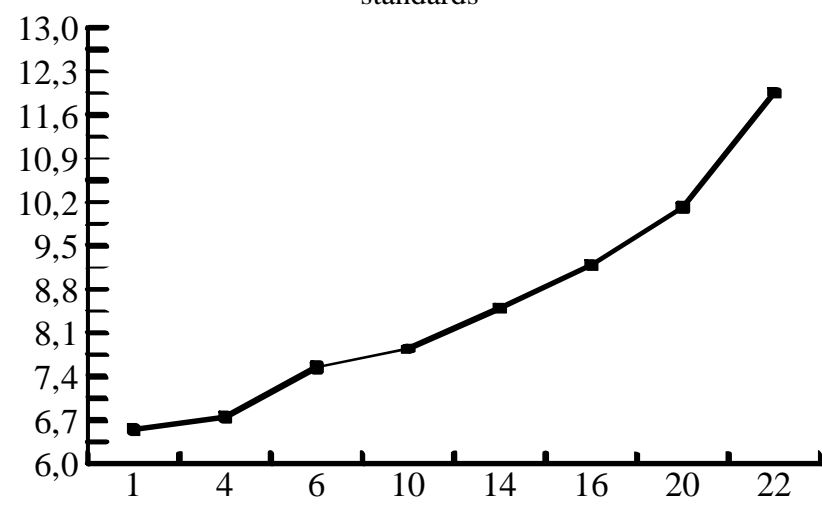

Figure. 9. Schedule time change detection of individual flat objects on the number of standards

As seen from the graph in Figure 8 maximum error rate of 10 etalons is $4 \%$, and at 20 and more reference error becomes zero.

\section{RECOGNITION OF INDIVIDAUL FLAT OBJECTS IN THEIR CONDITION IMPOSED}

This method is like any recognition method consists of two main phases - training and exam.

During the training phase, the following steps:

1. Generate imposed objects (let's call a complex object) of two flat objects. 10 flat objects are formed 55 pairs imposed on 2 sites. The creation of complex objects is to generate versions of the image of each individual flat object and their placement on the same field of view of the recognition system.

The imposition of any two types of objects in the field of view is determined by the terms of the marking:

- If the field of view of one connected component, the objects are superimposed or concern (zero overlay);

- If the sight of two (or more) of the connected components, the objects are not imposed.

2. Determination of the frequency of occurrence of a complex object with a specific percentage of the superposition of two objects.

3. Pre-treatment images of complex objects - the same pretreatment in the first recognition algorithm.

4. Formation of R-functions of a complex object. function similar to the formation of R-functions in the first algorithm recognition as a binary image of a complex object in the field is a separate object.

Figure 10, Figure 11 shows plots of the probability of correct recognition, and time for all 55 types of complex objects on the amount of standards.

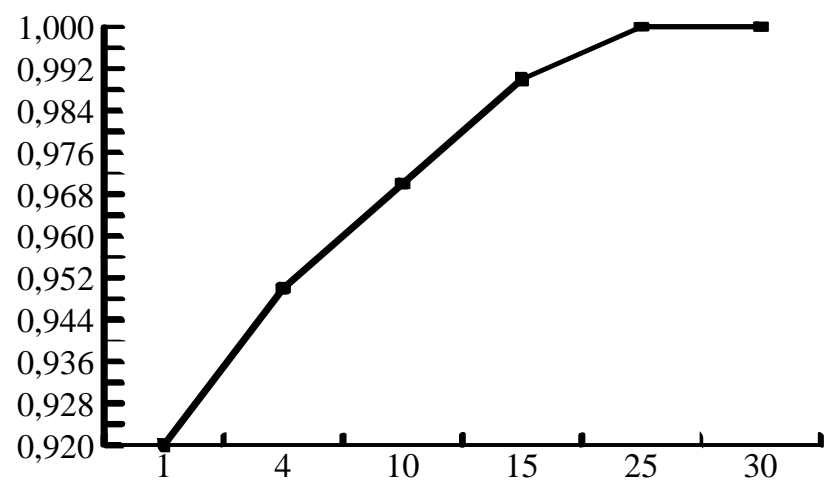

Figure. 10. A plot of the probability of correct recognition of complex object on the number of standards

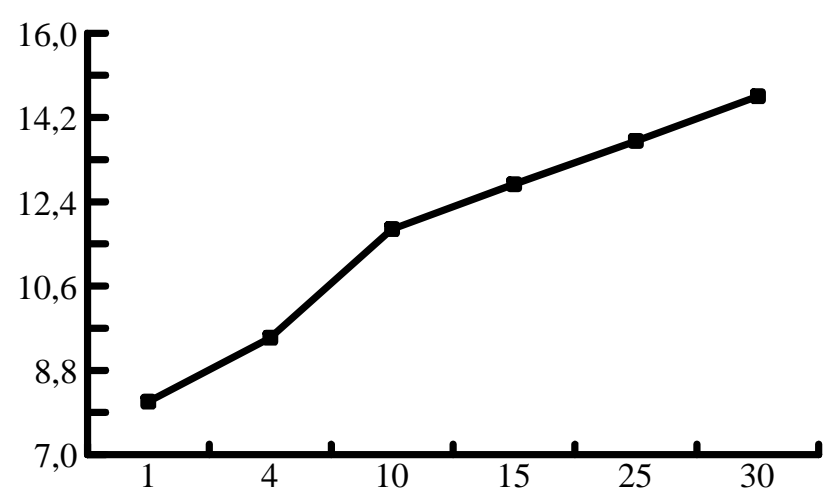

Figure. 11. Schedule change the time of a complex object recognition on the number of standards

Analysis of the results shows that at 25 measurement standards ensures $100 \%$ detection of all 110,000 options 55 pairs of complex objects. This means we can assume that the probability of error detection of any complex object using the first recognition algorithm is less than $1 \cdot 10^{-5}$.

Complex object recognition and identification of its facilities lets you know of any individual flat objects is a complex object. Now you need to determine what part of a complex object is one and the other separate flat object. This problem is solved by using the second pattern recognition algorithm separate flat objects in the following order:

1. Forming reference A-function initial 10 separate flat objects.

2. Generate A-feature detects a complex object.

3. A comparison of the reference function of a single flat object and A-function complex object.

A similar comparison of AE function of the second subject is an individual with all the A-functions of complex objects.

Figure. 12 shows a graph of the recognition accuracy, separate flat objects in each of the superimposed object 55 depending on the number of standards for each complex object. Figure 13 is a graph of change of probability of correct recognition of certain objects in the plane of complex objects when the number of standards from 1 to 14 . 


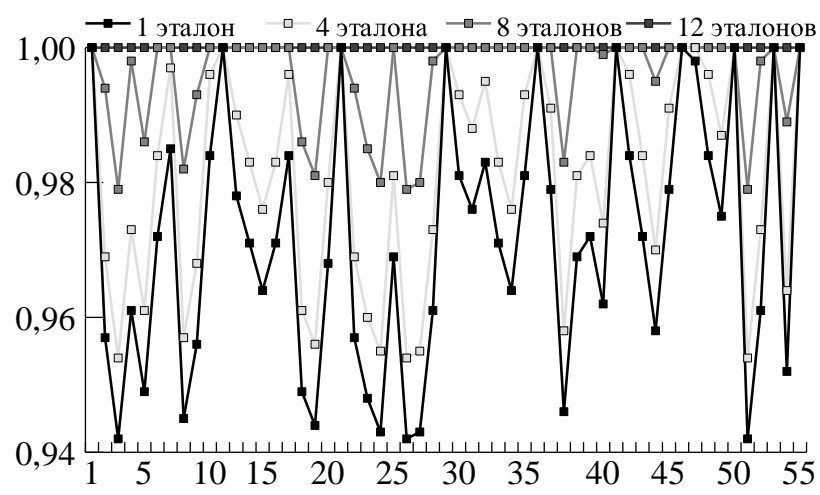

Figure. 12. Schedule changes in the probability of correct recognition of individual flat objects in each of the 55 complex objects with different number of reference

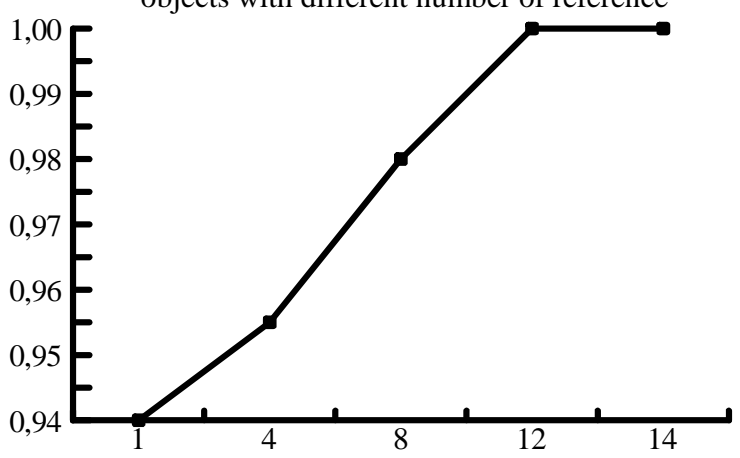

Figure. 13. A plot of the probability of correct recognition of individual flat objects in a complex object on the number of standards

Time graph of the recognition of the number of standards is shown in Figure 14.

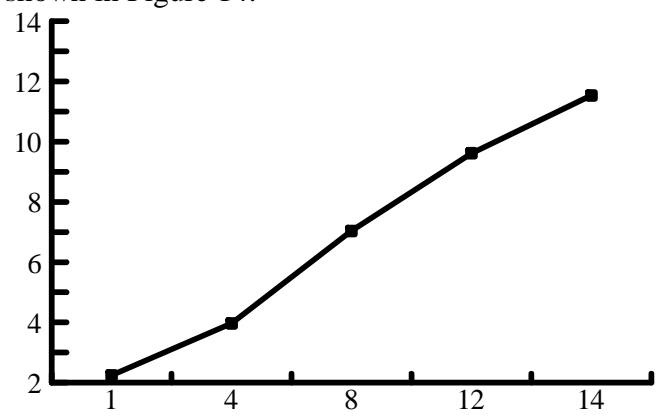

Figure. 14. A plot of the time of recognition of individual flat objects in a complex on the number of references to an object Analysis of the graphs in Figure. 12-14 shows that the maximum error rate of recognition of individual objects in the complex plane based on the A-function in a plurality of reference is: at 4 standard $-5.5 \%$; time $-3.968 \mathrm{~ms}$; etalons at 8 $2 \%$; time $-7.038 \mathrm{~ms}$; at 12 or more etalons - $0 \%$; time -9.615 ms.

\section{CONCLUSION}

1. R-algorithm provides for a small number of reference for each object and a small-time high accuracy of recognition of individual flat objects With it successfully solved the problem of determining the class of a complex object formed by the superposition of two separate flat objects.

2. A recognition algorithm allows individual flat objects, but with a large number of references to an object, and longer than the R-algorithm. Detection of individual objects in the complex plane is possible by using only A-algorithm.
3. The method of recognition of individual flat objects in a complex object, based on the R- and A-functions provides high recognition accuracy with a small number of reference as a complex object, and a separate flat objects.

\section{REFERENCES}

Sadykov, S.S., Savicheva, S.V. 2012. Pretreatment images of flat objects in vision systems. Instrument, 2, pp. 19-24.

Sadykov, S.S., Savicheva, S.V. 2012Evaluation methods recognition capabilitiestion of flat objects. Algorithms, methods, and data processing systems. 4 (22). pp. 110-119.

Sadykov, S.S., Savicheva, S.V. 2011. Identification of the actual plane of objects based on a single feature of their points of external circuits. Information Technology. 8. pp. 13-16.

Sadykov, S.S., Savicheva, S.V. 2012. Study imposing flat objects in sight STZ. Instrument. 2. pp. 14-19

Revised March 2015 